\title{
Further Development and Assessment of a Broadband Liner Optimization Process
}

\author{
Douglas M. Nark*and Michael G. Jones ${ }^{\dagger}$ \\ NASA Langley Research Center, Hampton, VA 23681-2199, U.S.A \\ Daniel L. Sutliff ${ }^{\ddagger}$ \\ NASA Glenn Research Center, Cleveland, OH 44212, U.S.A
}

\begin{abstract}
The utilization of advanced fan designs (including higher bypass ratios) and shorter engine nacelles has highlighted a need for increased fan noise reduction over a broader frequency range. Thus, improved broadband liner designs must account for these constraints and, where applicable, take advantage of advanced manufacturing techniques that have opened new possibilities for novel configurations. This work focuses on the use of an established broadband acoustic liner optimization process to design a variable-depth, multi-degree of freedom liner for a high speed fan. Specifically, in-duct attenuation predictions with a statistical source model are used to obtain optimum impedance spectra over the conditions of interest. The predicted optimum impedance information is then used with acoustic liner modeling tools to design a liner aimed at producing impedance spectra that most closely match the predicted optimum values. The multi-degree of freedom design is carried through design, fabrication, and testing. In-duct attenuation predictions compare well with measured data and the multi-degree of freedom liner is shown to outperform a more conventional liner over a range of flow conditions. These promising results provide further confidence in the design tools, as well as the enhancements made to the overall liner design process.
\end{abstract}

\section{Nomenclature}

$c$

$h_{i}$

$i$

$J_{n}$

$k$

$p$

$T_{i j}$

$u$

$W_{i}$ sound speed

characteristic distance (or height)

$\sqrt{-1}$

Bessel function of order $n$

free-space wave number $(k=\omega / c)$

acoustic pressure normalized by $\rho c^{2}$

transmission coefficient

acoustic particle velocity normalized by $c$

weighting values

\footnotetext{
* Senior Research Scientist, Structural Acoustics Branch, Research Directorate, AIAA Associate Fellow

${ }^{\dagger}$ Senior Research Scientist, Structural Acoustics Branch, Research Directorate, AIAA Associate Fellow

${ }^{\ddagger}$ Aerospace Engineer, Acoustics Branch, AIAA Associate Fellow
} 
Symbols:

$\begin{array}{ll}\beta & \text { admittance }(\beta=1 / \zeta) \\ \Gamma & \text { propagation constant } \\ \zeta & \text { acoustic impedance normalized by } \rho c(\zeta=\theta+i \chi) \\ \theta & \text { acoustic resistance normalized by } \rho c \\ \mu & \text { mean value } \\ \rho & \text { fluid density } \\ \sigma & \text { square root of the Prandtl number }(\sigma=\sqrt{P r}) \\ \chi & \text { acoustic reactance normalized by } \rho c \\ \Omega & \text { fraction of treated area } \\ \omega & \text { angular frequency }\end{array}$

Abbreviations:

CDL

LTF

CDUCT-LaRC

MDOF

Liner Test Facility

RPMc

Multi-Degree of Freedom

SDOF

Revolutions-Per-Minute (corrected)

TRL

Single-Degree of Freedom

Technology Readiness Level

\section{Introduction}

The ability to predict fan noise within complex aircraft nacelle geometries is invaluable in optimizing acoustic liner treatment. The utilization of advanced fan designs (including higher bypass ratios) and shorter engine nacelles has highlighted a need for increased fan noise reduction over a broad frequency range. Thus, improved broadband liner designs must account for these constraints and, where applicable, advantage may be taken of advanced manufacturing techniques that have opened new possibilities for novel configurations. With these observations in mind, the development and assessment of a broadband acoustic liner optimization process has been pursued through a series of design studies. ${ }^{1 / 3}$ In this design process, an acoustic duct propagation code is used to predict optimum impedance spectra over a number of frequencies and flow conditions. Acoustic liner modeling tools are then used to identify geometric liner parameters (within manufacturing constraints) necessary to produce impedance spectra that most closely match the predicted optimum values. The resultant impedance values are then used with the propagation code to produce predicted attenuation spectra. Iteration between the prediction and design stages is possible to allow refinement of the liner design.

To gain confidence in the tools used within the broadband liner design process, a series of multi-degree of freedom (MDOF) liner design studies at increasing technology readiness levels (TRL) has been conducted as the overall optimization methodology has been enhanced. Initial liner designs targeting the NASA Langley Liner Technology Facility ${ }^{45}$ (LTF) resulted in predicted optimum impedance spectra consistent with those obtained using higher-fidelity, more computationally intensive models. Subsequent design and testing in the NASA Glenn Advanced Noise Control Fan (ANCF) Rig ${ }^{3 / 6}$ provided further confidence in the design process. Based on these results, fabrication and testing of an MDOF liner design for a high speed fan was pursued and is the focus of this work. The latest test configuration and associated measurements are described in Section II] The computational approach, impedance modeling, and liner design process are then presented in more detail in Sections III, IV, and $\mathrm{V}$, respectively. Evaluation of liner performance and comparison with predictions are provided in Section $\mathrm{VI}$. Finally, concluding remarks regarding some of the more significant results and further areas of interest are presented in Section VII

\section{Test Facility and Acoustic Measurements}

Under a pair of Space Act Agreements between NASA and Honeywell Aerospace, a model scale (22-inch diameter fan) acoustic wind tunnel tes ${ }^{7}$ was carried out in the NASA Glenn 9- $\mathrm{ft} x$ 15-ft Low Speed Wind Tunnel (9x15). The NASA 9x15, a schematic of which is shown in Figure 1 is an open-loop, continuous-flow, anechoic wind tunnel facility. Acoustic measurements are acquired using a traversing microphone placed at sideline locations 89.3 inches 
(approximately four fan diameters) from the fan axis. Data are acquired at 48 positions in 2.5-degree intervals starting at $27^{\circ}$ from the fan axis, with $0^{\circ}$ being the forward position upstream of the fan. Additionally, to obtain more angular coverage in the aft quadrant, three fixed microphone probes are placed in the rear of the test section. Together, the traversing probe and the fixed microphones result in 51 possible measurement locations that cover sideline geometric angles ranging from $27^{\circ}$ to $160^{\circ}$.

In addition to the sideline acoustic measurements, a rotating rake measurement system ${ }^{8 / 9}$ was used to acquire modal distributions of the acoustic field in the fan duct 10 Developed and implemented by NASA Glenn Research Center in the 1990s to measure turbofan duct acoustic modes, the system is a continuously rotating radial microphone rake that is inserted into either the inlet or the fan exhaust ducts. The rotating rake provides a map of the acoustic duct modes present inside the nacelle and the technique has been used on a variety of test articles ranging from a low-speed, concept test rig to a full scale production turbofan engine. The key concept behind the rotating rake technique is that by rotating the rake at a fraction of the fan shaft rotational speed (e.g., 1:200 speed ratio), a Doppler shift is imparted to the duct spinning modes that is based on the mode number and the selected speed ratio. The acoustic pressure profile may therefore be separated into modes and the associated acoustic power level (PWL) computed at the rotating rake axial station. While further details on the rotating rake measurements are provided in a companion paper, $\frac{10}{10}$ the resultant measurement of in-duct attenuation is used to assess predictions generated during the liner design process.

Due to the proprietary nature of the test fan hardware, the actual test geometry cannot be presented. However, a general understanding of the bypass duct geometry and liner locations may be obtained from the representative geometry shown in Figure 2. The MDOF design includes three distinct liner zones (labeled Nacelle Liner, Hub Liner-1, and Hub Liner-2) for which the individual impedances were optimized. In addition to the MDOF liner, a single-degree of freedom (SDOF) liner designed by Honeywell was also tested. This SDOF liner consisted of only two zones with differing depths on the inner and outer walls. Similar to the MDOF liner, the intent of this design was to achieve more broadband attenuation, but with a traditional implementation. While not a main aspect if this paper, results from the SDOF will be presented in Section VI to illustrate the validity of the prediction tools, as well as the relative performance of the SDOF and MDOF liners.

\section{Optimum Impedance Prediction}

For the current investigation, duct propagation and radiation predictions are conducted using the CDUCT-LaRC (CDL) code. This code calculates the propagation of a given acoustic source ahead of the fan face or aft of the exhaust guide vanes in the inlet or exhaust ducts, respectively. Subsequent to the propagation calculations, the code has the capability of computing the noise radiation field outside the duct. The three-dimensional duct may include acoustic treatment (possibly circumferentially and radially segmented) and incorporate struts/bifurcations. All of the modules that currently make up the CDL framework have been discussed previously 11 and are utilized in this study. However, this discussion will focus on the propagation and radiation modules, as they are most pertinent to this investigation.

The duct propagation module is based on the CDUCT code developed by Dougherty ${ }^{12} \mid 13$ and extended by Lan. 14 This code utilizes a parabolic approximation to the convected Helmholtz equation and offers a computationally efficient model that accounts for the complexities of fully three-dimensional nacelle configurations. The CDL code has been extended ${ }^{11}$ to support multi-block propagation calculations. The grid connectivity is determined and data is transferred from upstream to downstream blocks without user intervention. Results of the propagation module include the acoustic potential or pressure within the duct, which may be utilized by the radiation module for acoustic radiation calculations.

The duct radiation model is based on the Ffowcs Williams-Hawkings (FW-H) equation with a permeable data surface ${ }^{[15]}$ Based on the background flow conditions and propagation solution, this module calculates the radiated acoustic pressure at various observer locations. Currently, the data surface is taken to be the nacelle inlet or exhaust plane for inlet or aft-fan cases, respectively. However, provisions have also been made to attempt to account for the effects of the shear layer on the radiation.

While CDL can accept arbitrary source specification (i.e., it is not a modal code), it is convenient to specify the acoustic source distribution in terms of duct modes. For situations in which the source pressure is available, this greatly simplifies conversion to the required acoustic potential. However, when source information is not available, an assumption on the source description must be made. This is generally the case and the approach taken here follows that described by Zlavog and Eversman 16,17 in a series of statistical studies into the effects of randomized modal source power and/or phase on attenuation in lined ducts. In this study, the source modal powers (and hence, amplitudes) and modal phases are allowed to vary randomly and independently. Thus, with equal probability of occurrence, the amplitude and phase for each cut-on source mode may take any value in the intervals from 0 to 1 and 0 to $2 \pi$, 
respectively. This uncertainty in source specification will undoubtedly result in uncertainty in the predicted sound levels. However, as discussed by Zlavog and Eversman, $\frac{16,17}{17}$ for the case of all propagating circumferential and radial acoustic modes with random modal power and phase, transmitted power appears to be normally distributed. In their studies, this also produced statistical distributions with the smallest standard deviation. Therefore, as discussed in a previous CDL study, ${ }^{18}$ Student's t-distribution is used to statistically analyze the predicted attenuation results. For each configuration and power setting, 11 simulations were performed to produce a sample population from which statistical information can be inferred.

As with previous liner optimization studies,,$\sqrt{1 / 3}$ in-duct attenuation was chosen as the cost function for the impedance optimization. Thus, the optimum impedance spectrum was predicted using the propagation module of the CDL code with the aforementioned source model. A constant-azimuth portion of the computational domain for these predictions is shown as a green cross-hatched region in Figure 2. Previously, the maximum in-duct attenuation was obtained by plotting predicted mean attenuation over a specified normalized resistance range of $0<\theta \leq 5$ and a normalized reactance range of $-5 \leq \chi \leq 5$. Preliminary estimates were obtained using a coarse grid over the full impedance domain. A fine grid over a sub-domain encompassing the preliminary value was then used to obtain a refined optimum impedance prediction. It is evident that this approach involves a large number of propagation calculations. To alleviate this issue, the current study coupled the CDL calculations with optimization routines. This was accomplished by using the Python programming language (http://www.python.org) to "wrapper" the CDL calculations. The use of the Python scripts allows access to the full set of tools available in the optimization package of the Scipy ${ }^{19}$ library. Thus, predicted optimum impedance spectra (over a larger design space encompassing $0<\theta \leq 5$ and $-10 \leq \chi \leq 10$ ) were obtained with a large reduction in computational effort. Optimum impedance predictions were obtained for the one-third octave center frequencies ranging from $2000 \mathrm{~Hz}$ to $8000 \mathrm{~Hz}$ at flow conditions $(61 \%, 75 \%$, and 93\% RPMc) representative of standard noise certification points.

\section{Impedance Prediction Model}

The impedance prediction model used in this study combines two models presented in an earlier paper. ${ }^{20}$ The first is a transmission line mode ${ }^{21}$ that assumes acoustic wave propagation through each layer of the liner (see Fig. 3), and the second is a lumped element mode ${ }^{\sqrt{22}-24}$ used to compute the impedance change across perforates. The normalized surface impedance spectra presented by each individual chamber of the liner are computed separately, and are then combined to determine an effective surface impedance spectrum that is assumed uniform across the liner surface.

\section{A. Single Chamber Computations}

The transmission line model for predicting the surface acoustic impedance of an individual liner chamber is briefly described herein with the aid of Fig. 3. This figure provides a sketch of a single chamber of a liner with two air gaps (sections with heights of $h_{1}$ and $h_{3}$ ) and two perforate sheets (thicknesses of $h_{2}$ and $h_{4}$ ). The thickness of each perforate sheet is exaggerated to demonstrate distinct features of the models used in this study. This model treats each unique portion of the liner chamber separately via a transmission line computation. In the following discussion, these unique portions are treated as individual "computational layers."

The transmission line computation is initiated by assuming the backplate at $x_{0}$ to be rigid and impermeable. The normalized acoustic pressure and particle velocity are given as

$$
\left(\begin{array}{l}
p_{0} \\
u_{0}
\end{array}\right)=\left(\begin{array}{l}
1 \\
0
\end{array}\right)
$$

which yields an infinite impedance at the backplate of the liner. All acoustic pressures and particle velocities are normalized by $\rho c^{2}$ and $c$, respectively, where the density of the air and the speed of sound are for the prevailing static pressure and air temperature. Correspondingly, impedances are normalized by the characteristic impedance of air, $\rho c$. Changes in the acoustic pressure and particle velocity across a computational layer are computed via

$$
\left(\begin{array}{l}
p_{n+1} \\
u_{n+1}
\end{array}\right)=\left(\begin{array}{ll}
T_{11} & T_{12} \\
T_{21} & T_{22}
\end{array}\right)\left(\begin{array}{l}
p_{n} \\
u_{n}
\end{array}\right)
$$

where the transmission coefficients $\left(T_{11}, T_{12}, T_{21}\right.$ and $\left.T_{22}\right)$ depend on the modeling approach and the type of layer being considered.

For open layers (air gaps), the transmission coefficients are given by 


$$
T_{11}=T_{22}=\cosh (k \Gamma h) ; T_{12}=\zeta_{c} \sinh (k \Gamma h) ; T_{21}=\zeta_{c}^{-1} \sinh (k \Gamma h)
$$

where $k=\omega / c$ is the free-space wavenumber, $\omega$ is the angular frequency, and $h$ is the layer thickness. The propagation constant, $\Gamma$, and characteristic impedance, $\zeta_{c}$, are computed as

$$
\Gamma=\sqrt{\frac{J_{0}\left(i^{3 / 2} s\right)}{J_{2}\left(i^{3 / 2} s\right)}} \sqrt{\frac{\gamma}{n_{\Gamma}}} ; \quad \zeta_{c}=\frac{-i}{\Gamma} \frac{J_{0}\left(i^{3 / 2} s\right)}{J_{2}\left(i^{3 / 2} s\right)}
$$

where

$$
n_{\Gamma}=\left[1+\frac{\gamma-1}{\gamma} \frac{J_{0}\left(i^{3 / 2} \sigma s\right)}{J_{0}\left(i^{3 / 2} \sigma s\right)}\right]^{-1}
$$

$i=\sqrt{-1}$ is the imaginary unit, $s=\left(d_{c} / 2\right) \sqrt{\rho_{s} \omega / \mu}$ is the shear wave number, and $d_{c}, \rho_{s}, \omega, \mu, \sigma$ and $\gamma$ are the channel diameter, static density, angular frequency, coefficient of viscosity, square root of the Prandtl number and specific heat ratio, respectively. Note that if a liner chamber only contains a single open computational layer (cavity with no facesheet), the normalized surface impedance for that chamber is computed as

$$
\zeta_{c h}=\frac{p_{1}}{u_{1}}
$$

For those cases where the liner chamber contains more than one computational layer, the acoustic pressure and particle velocity computed at the top of the first computational layer $\left(p_{1}\right.$ and $\left.u_{1}\right)$ are input back into Eq. 2 to compute the impedance change across the next computational layer.

Two approaches are considered for computing the change in impedance across an embedded septum, assumed to consist of either a perforated sheet or a wire mesh. The first assumes the septum to be a purely resistive lumped element that is too thin to support wave propagation. For this approach, the transmission coefficients for Eq. 2 are given by

$$
T_{11}=T_{22}=1 ; T_{12}=\frac{R_{f}}{\rho c} ; T_{21}=0
$$

where the normalized DC flow resistance, $R_{f} / \rho c$, across the perforated sheet is often estimated from a lumped element, semi-empirical model (e.g., the Two-Parameter Impedance Prediction Model23).

The second approach assumes the perforated sheet to be sufficiently thick to support wave propagation. Figure 3 is particularly useful to describe this approach. It is assumed that Eqs. 2 and 3 have been used to determine the acoustic pressure and particle velocity ( $p_{1 a}$ and $u_{1 a}$ ) at the top of the lower air gap. The acoustic pressure and acoustic mass flow are assumed to be constant across the $\{1 a, 1 b\}$ interface, such that

$$
p_{1 b}=p_{1 a} ; \quad N S_{1 b} u_{1 b}=S_{1 a} u_{1 a}
$$

where $N$ is the number of orifices ( 3 in this example) connected to a single air cavity, $S_{1 a}$ is the cross-sectional area at the top of the air cavity (denoted by the long dashed line at the top of the cavity) and $S_{1 b}$ is the cross-sectional area within a single perforate orifice. The wave propagation within the single orifice is then computed using Eqs. 2 and 3 . where the orifice diameter is used as the "channel diameter." Again, the acoustic pressure and acoustic mass flow are assumed to be constant across the $\{2 a, 2 b\}$ interface, such that

$$
p_{2 b}=p_{2 a} ; \quad S_{2 b} u_{2 b}=N S_{2 a} u_{2 a}
$$

Regardless of which approach is used to model embedded septa (for those configurations that contain them), the process described above is repeated until the acoustic pressure and particle velocity are determined at $x_{3}$ (the plane just below the perforated facesheet). The normalized impedance at $x_{3}$ is then given by

$$
\zeta_{n-1}=\frac{p_{n-1}}{u_{n-1}}
$$

( $n=4$ for the example given in Fig. 3). The Two-Parameter Impedance Prediction Model is then used to predict the impedance change across the perforated facesheet, such that the effects of grazing flow over the surface of the liner can be included. The resultant normalized surface impedance for the chamber (at the plane denoted by the dashed line with endpoints $A$ and $B$ ) is given by 


$$
\zeta_{c h}=\frac{p_{n}}{u_{n}}
$$

where $n$ represents the number of computational layers in the chamber.

\section{B. Liner Surface Impedance Computation}

The surface impedance spectra of the individual chambers are combined to compute the effective impedance across the liner surface. For this computation, it is preferable to use acoustic admittance $\left(\beta_{c h}=1 / \zeta_{c h}\right)$. The effective admittance across a selected extent of liner surface is given by

$$
\beta_{s}=\Omega \sum_{i=1}^{N_{c h}} \beta_{c h}
$$

where $N_{c h}$ represents the number of chambers that combine to form the liner and $\Omega$ is the fraction of the surface that is comprised of this group of chambers. The uniform, effective, surface impedance of the liner is then given by $\zeta_{s}=1 / \beta_{s}$.

\section{Liner Design}

As indicated above, the CDL propagation code was used to determine target optimum impedance values within the duct. The efficiency of this process allows for parallel-element, variable-impedance, broadband liner concepts at various technology readiness levels (TRL) to be considered for implementation. To achieve broadband performance, the liner consisted of a multi-layer configuration incorporating septa (or "mesh-caps") embedded into a honeycomb core ${ }^{20}$ This concept allows the acoustic liner to be customized such that the surface impedance of each individual cell is independently controlled. This is achieved by the combination of parameters used to set the impedance in each cell. The various cells are then arranged into a grid pattern of different mesh-cap depths or resistances within the acoustic panel to achieve a desired distributed impedance, as illustrated in Figure 4 Clearly, manufacturing constraints (e.g., liner geometric parameters such as porosity and core depth) are key ingredients in this modeling phase, and must be taken into account to design acoustic liners that can be realistically achieved. In this study, the honeycomb cells were restricted to contain no more than one mesh-cap. The mesh-cap depth, as well as its DC flow resistance, were allowed to vary from cell to cell. The CDL code is used to predict optimum impedance values, $\zeta_{\text {opt }}\left(f_{i}\right)$, at selected frequencies, $f_{i}$, for the given flow speed. The liner modeling tools are then used to obtain design impedance values, $\zeta_{\text {pred }}\left(f_{i}\right)$, that minimize the function

$$
F=\left\{\sum_{i=1}^{7} W_{i}\left(\zeta_{\text {opt }}\left(f_{i}\right)-\zeta_{\text {pred }}\left(f_{i}\right)\right)\left(\zeta_{\text {opt }}\left(f_{i}\right)-\zeta_{\text {pred }}\left(f_{i}\right)\right)^{*}\right\}^{0.5} .
$$

Here, the values $W_{i}$ are weighting values that may be used to assign increased significance to a selected number of frequencies and/or flow conditions and $N$ is the total number of frequency/flow condition combinations considered. Note that with $W_{i}=1$ for all $N$, equation 13 reduces to the $L_{2}$ norm of the difference between the predicted optimum and the design impedance values.

Based on the above cost function, a MDOF liner was designed and manufactured. As seen in Figure 2 the MDOF liner consisted of three distinct zones, the overall depths of which were chosen based on the space available in the inner and outer walls of the fan rig. In the outer wall (Nacelle Liner), the maximum depth was approximately 1 in $(2.54 \mathrm{~cm})$ over the entire length of the treated section. For the inner wall, the maximum depth was 0.76 in $(1.93 \mathrm{~cm})$ over the forward portion (Hub Liner-1), and 0.36 in $(0.91 \mathrm{~cm}$ ) over the aft portion (Hub Liner-2). Clearly, those portions for which the available depth was larger allowed for a wider range of septa depths and, hence, more options in the liner design. A $16 \%$ porosity facesheet with a thickness of 0.04 in $(0.10 \mathrm{~cm})$ and a $0.1 \mathrm{in}(0.25 \mathrm{~cm})$ backplate were selected for all zones. The DC flow resistance of the mesh-cap material within a given cell was allowed to vary from 400 to 1200 in steps of 100 MKS Rayls. The ability to mimic "shallower" cells (depths less than that of the liner core) was included in the design by additionally allowing septa having a DC flow resistance of 4150 MKS Rayls. The depth of the septa was also allowed to vary nominally between 0.05 in $(0.13 \mathrm{~cm})$ from the backplate or facesheet in steps of 0.1 in $(0.25 \mathrm{~cm})$. Finally, allowance was made for cells with no septa, to achieve a maximum-depth single-layer cell.

The aforementioned liner modeling approach (Section V) was used to generate a database of impedance values based on the given range of liner parameters. In contrast to the optimization methods used in Section IV] this aspect of the liner modeling has not been "wrappered" to take advantage of more sophisticated optimization algorithms. 
Therefore, for each liner zone, a "brute-force" optimization process was used to determine the best combination of geometric parameters to achieve the desired impedance values. Due to the number of possible combinations, a fourcell pattern of mesh cap resistance and insertion depth (see Figure 4) was initially generated. Individual cells were then added (one at a time) to ultimately design an eight-cell pattern that was replicated over the selected treatment area.

Comparison of the predicted optimum and design impedances are shown in Figure 5 . It can be seen that the character of the target impedance (relatively low resistance at lower frequencies and decrease in reactance with increasing frequency) makes it difficult to match with a physically-realizable liner, particularly at the lower frequencies. The design resistance values are generally above the optimum values and the $-\cot (k d)$ behavior evident in the design reactance spectra is generally counter to the optimum reactance trend. However, in light of the amount of available space (particularly in the inner wall) the results are promising. As eluded to previously, the comparison between optimum and MDOF impedance spectra was improved for those liner zones which allowed for a wider range of septa depths and, hence, more variability in the liner design. It should also be noted that efforts are underway to replace the "brute-force" optimization process with the Scipy $\frac{19}{19}$ optimization tools used in predicting the optimum impedance spectra (Section III). This enhanced capability will provide increased efficiency, as well as access to a much wider design space in matching the target impedance spectra.

\section{Results and Discussion}

In order to provide further confidence in the liner design process and evaluate liner performance, comparisons are made between the predicted and measured attenuation values. Specifically, in-duct attenuation was chosen for this study, as the MDOF liner design is based on this quantity (investigation of the far-field data is reserved for future work). Due to the proprietary nature of the SDOF liner, details of the design process used by Honeywell are not discussed. However, the relative performance results are not subject to this constraint and are therefore presented in Figure 6 at three fan speeds representative of standard certification points. The prediction values, shown as vertical bars, are based on the liner modeling presented in Section IV and are therefore presented at the seven one-third octave band center frequencies considered in the optimization. Due to the statistical nature of the source, error bars representing the $95 \%$ confidence intervals for the mean attenuation are also included. The measured values, shown as red circles, represent insertion loss obtained from the aforementioned rotating rake data which, based on previous experience, are expected to have a measurement error of $+/-1 \mathrm{~dB} \cdot{ }^{6}$ Measurements were made at frequencies corresponding to the blade passage frequency (BPF) and its first two harmonics. Note that for the highest fan speed, 3BPF fell well outside the prediction range and therefore was not included in the comparison. Overall, the predictions compare well with the measured values. The trends are well captured and the in-duct attenuation values are generally well matched. As mentioned previously, the SDOF results are included to further illustrate the validity of the prediction tools, as well as the relative performance of the SDOF and MDOF liners. As the MDOF liner was the focus of this work, corresponding results are presented in Figure 7. The predicted values are represented in the same way as the SDOF results. However, to distinguish from the previous figures, the measured values are presented as blue circles. Again, the predicted and measured quantities compare well. The trends are well captured and the predicted confidence intervals encompass the measurements over the range of frequencies considered. In addition to providing further confidence in the design process, Figures 6 7 7 also afford the opportunity to evaluate the relative performance of the liners. This is discussed in detail, and further evidence is provided, in the companion paper describing the rotating rake testing ${ }^{10}$ However, it can be seen that the MDOF liner outperformed the SDOF liner over the frequency range considered.

\section{Concluding Remarks}

This paper describes the extension of a broadband acoustic liner optimization and design process to a high-speed fan. Based on in-duct attenuation predictions, a variable-depth, multi-degree of freedom (MDOF) liner was designed, fabricated, and tested. Comparisons of in-duct attenuation results showed the predictions to match the trends and overall levels of the measured data well over a range of flow conditions. As expected, the MDOF liner was shown to outperform the SDOF liner over the range of frequencies considered. These promising results provide further confidence in the design tools, as well as the enhancements made to the overall liner design process. Efforts are underway to replace the "brute-force" approach currently used in optimizing the liner geometric configurations. This enhancement will increase efficiency and provide a much wider design space in matching the target impedance spectra. This work presented an excellent opportunity to further evaluate the broadband liner design tools and illustrated the value of the design process in concurrently evaluating various liner designs (i.e., SDOF, MDOF, etc.) and their relative 
costs/benefits.

\section{Acknowledgments}

The authors wish to thank Dr. Chris Miller for his assistance with regard to the 9'x15' testing. The contributions of Earl Ayle, Fumitaka Ichihashi, and Clark Smith of Hexcel Corporation were also key to the MDOF liner fabrication. This research was funded by the Advanced Air Transport Technology (AATT) Project of NASA's Advanced Air Vehicles Program (AAVP).

\section{References}

${ }^{1}$ Nark, D. M. and Jones, M. G., "Broadband Liner Optimization for the Source Diagnostic Test Fan,” AIAA Paper 2012-2195, 2012.

${ }^{2}$ Nark, D. M., Jones, M. G., Sutliff, D. L., Ayle, E., and Ichihashi, F., "Improved Broadband Liner Optimization Applied to the Advanced Noise Control Fan," AIAA Paper 2014-3103, 2014.

${ }^{3}$ Nark, D. M., Jones, M. G., and Sutliff, D. L., "Modeling of Broadband Liners Applied to the Advanced Noise Control Fan," AIAA Paper 2015-2693, 2015.

${ }^{4}$ Gerhold, C. H., Brown, M. C., and Jones, M. G., "Segmented Liner to Control Mode Scattering," AIAA Paper 2013-2078, 2013.

${ }^{5}$ Jones, M. G., Watson, W. R., and June, J. C., "Optimization of Microphone Locations for Acoustic Liner Impedance Eduction,” AIAA Paper 2015-3271, 2015.

${ }^{6}$ Sutliff, D. L., Jones, M. G., and Nark, D. M., "In-Duct and Far-field Experimental Measurements from the ANCF for the Purpose of Improved Broadband Liner Optimization,” AIAA Paper 2014-3231, 2014.

${ }^{7}$ Miller, C. J., Stephens, D. B., Sutliff, D. L., and Dussling, J. J., “Acoustic Testing of a High Tip Speed Fan with Bypass-Duct Liners,” NASA TM 2015-xxxxx, 2015.

${ }^{8}$ Cicon, D. E., Sofrin, T. G., and Mathews, D. E., "Investigation of a Continuously Traversing Microphone System for Mode Measurement," NASA CR 168040, November 1982.

${ }^{9}$ Sutliff, D. L., "Turbofan Duct Mode Measurements Using a Continuously Rotating Microphone Rake," International Journal of Aeroacoustics, Vol. 6, No. 2, 2007, pp. 147-170.

${ }^{10}$ Sutliff, D. L., Nark, D. M., and Jones, M. G., "Efficacy of a Multiple Degree of Freedom Acoustic Liner Installed in the Bypass of a Scale Model High Speed Fan," AIAA Paper 2016-xxxx, 2016.

${ }^{11}$ Nark, D. M., Farassat, F., Pope, D. S., and Vatsa, V., "The Development of the Ducted Fan Noise Propagation and Radiation Code CDUCTLaRC," AIAA Paper 2003-3242, 2003.

${ }^{12}$ Dougherty, R. P., “A Wave-Splitting Technique for Nacelle Acoustic Propagation,” AIAA Paper 97-1652, 1997.

${ }^{13}$ Dougherty, R. P., "A Parabolic Approximation for Flow Effects on Sound Propagation in Nonuniform, Softwall, Ducts," AIAA Paper 99$1822,1999$.

${ }^{14}$ Lan, J. H., “Turbofan Duct Propagation Model,” NASA CR 2001-211245, 2001.

${ }^{15}$ Brentner, K. B. and Farassat, F., "Analytical Comparison of the Acoustic Analogy and Kirchhoff Formulation for Moving Surfaces," AIAA Journal, Vol. 36, No. 8, 1998, pp. 1379-1386.

${ }^{16}$ Zlavog, G. and Eversman, W., "Source effects on attenuation in lined ducts. Part I: A statistically based computational approach," Journal of Sound and Vibration, Vol. 307, No. 1-2, 2007, pp. 113-138.

${ }^{17}$ Zlavog, G. and Eversman, W., "Source effects on attenuation in lined ducts. Part II: Statistical properties," Journal of Sound and Vibration, Vol. 307, No. 1-2, 2007, pp. 139-151.

${ }^{18}$ Nark, D. M., “Assessment of Radiated Fan Noise Prediction Capabilities using Static Engine Test Data," AIAA Paper 2011-2807, 2011.

${ }^{19}$ Jones, E., Oliphant, T., Peterson, P., et al., "SciPy: Open source scientific tools for Python,” 2001 -.

${ }^{20}$ Jones, M. G., Howerton, B. M., and Ayle, E., "Evaluation of Parallel-Element, Variable-Impedance, Broadband Acoustic Liner Concepts," AIAA Paper 2012-2194, 2012.

${ }^{21}$ Parrott, T. L. and Jones, M. G., "Parallel-Element Liner Impedances for Improved Absorption of Broadband Sound in Ducts," Noise Control Engineering Journal, Vol. 43, No. 6, 1995, pp. 183-195.

${ }^{22}$ Motsinger, R. E. and Kraft, R. E., "Design and Performance of Duct Acoustic Treatment: Aeroacoustics of Flight Vehicles; Chapter 14, Vol. 2: Noise Control," NASA RP 1258, August 1991.

${ }^{23}$ Jones, M. G., Parrott, T. L., and Watson, W. R., "Uncertainty and Sensitivity Analyses of a Two-Parameter Impedance Prediction Model," AIAA Paper 2008-2928, 2008.

${ }^{24}$ Parrott, T. L. and Jones, M. G., "Assessment of NASA's Aircraft Noise Prediction Capability, Chapter 6: Uncertainty in Acoustic Liner Impedance Measurement and Prediction," NASA TP 2102-215653, July 2012.

${ }^{25}$ Dittmar, J. H., Elliott, D. M., and Fite, E. B., “The Noise of a Forward Swept Fan,” NASA TM 2003-212208, 2003. 


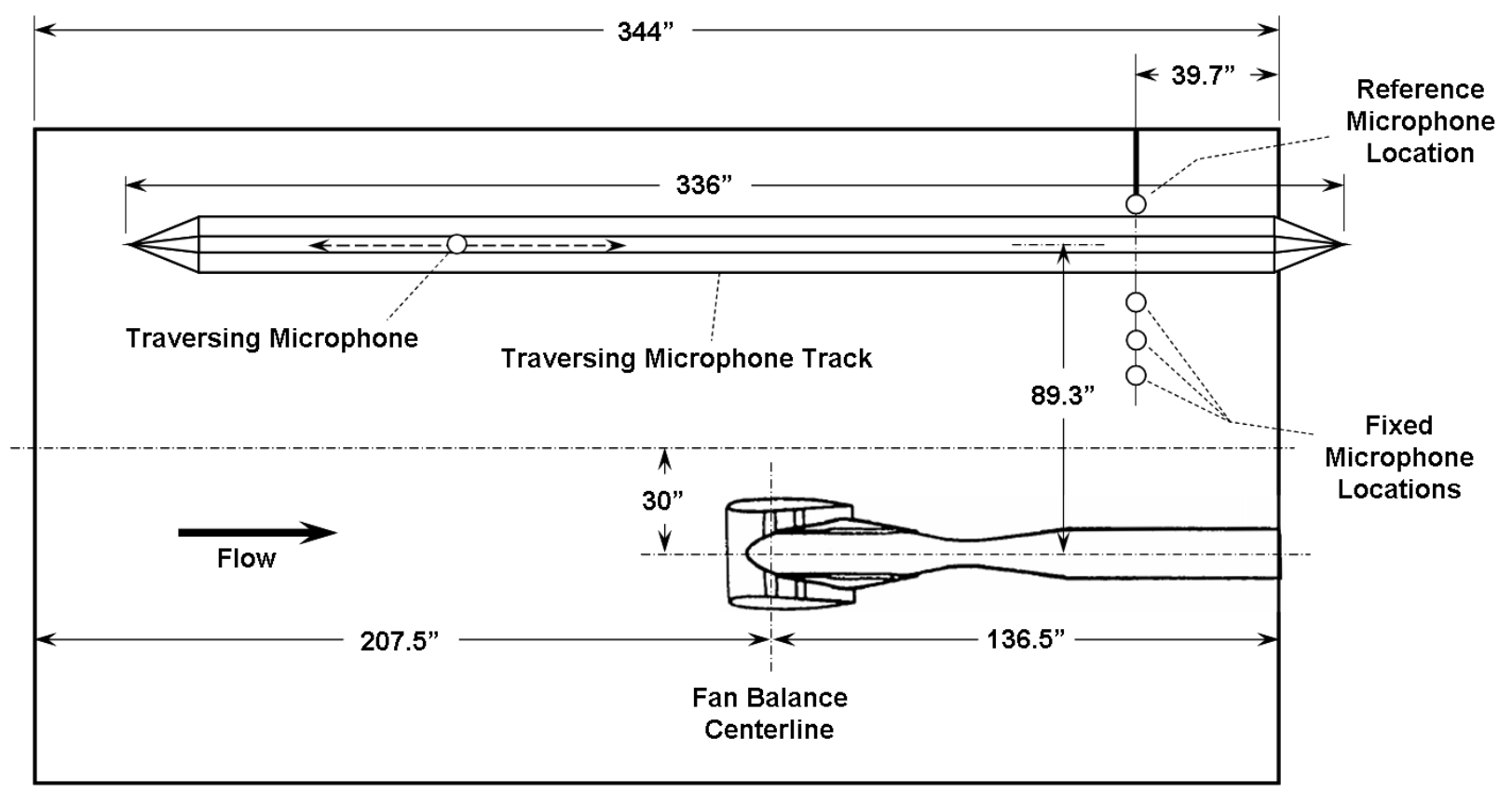

Figure 1: Top view of the 9' $\times 15$ ' test section illustrating model fan installation, as well as traversing microphone and three fixed aft microphones.

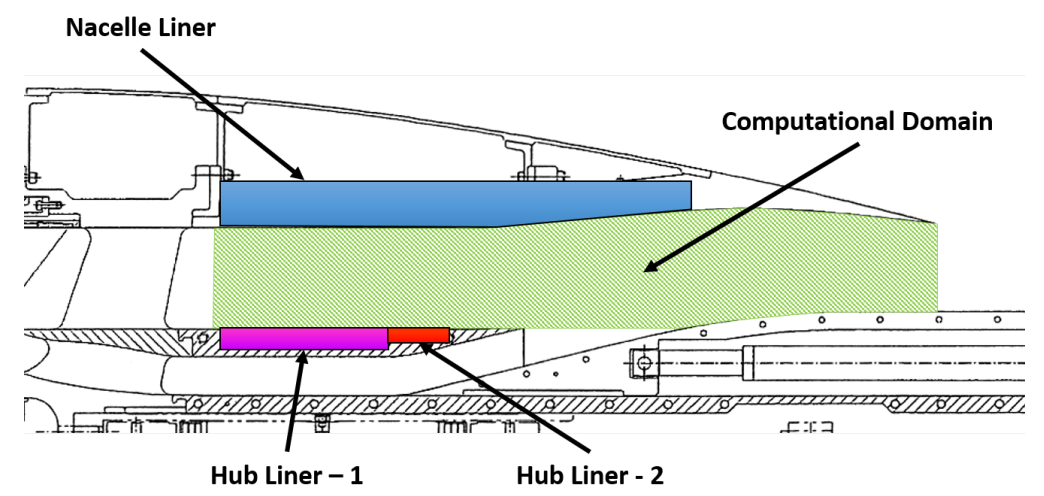

Figure 2: Representative bypass duct geometry.25

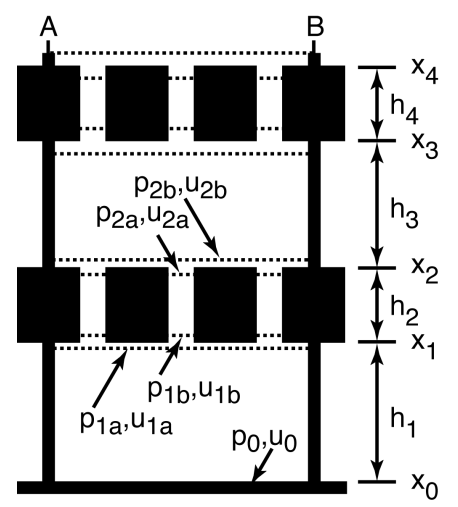

Figure 3: Sketch of single chamber of a liner with key parameters for transmission line impedance prediction model. 


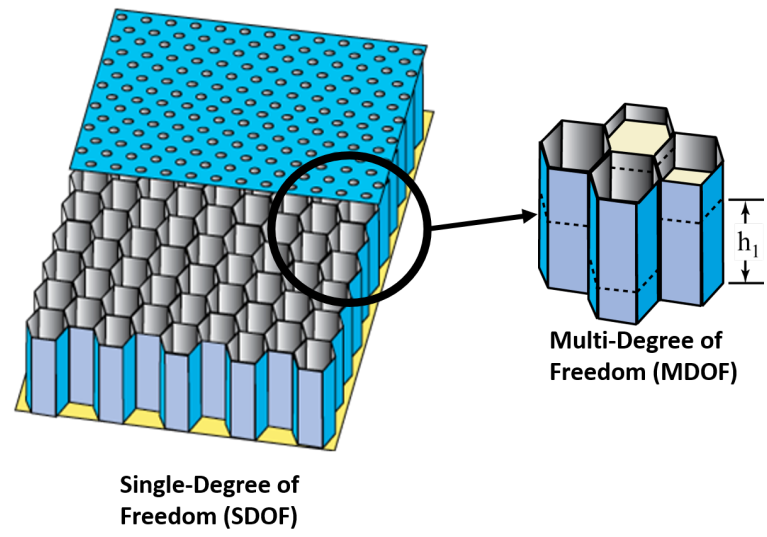

Figure 4: Parent single layer liner (SDOF) and resultant two-layer liner (MDOF) configurations via mesh-cap insertion.

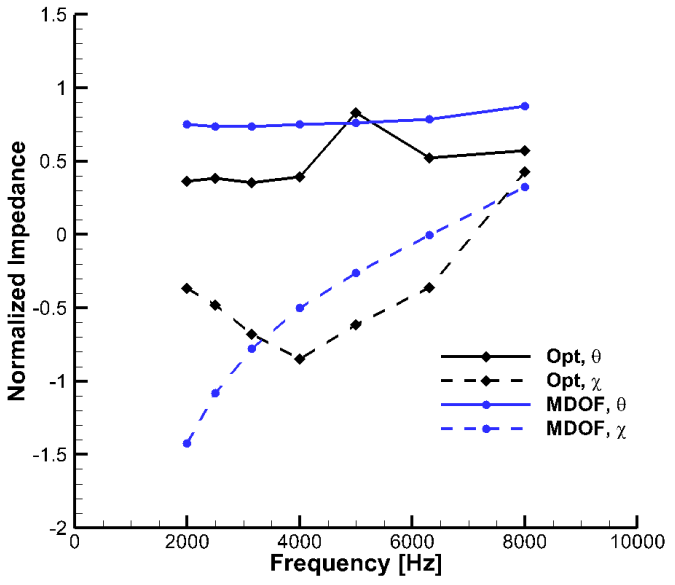

(a) Hub Liner - 1 .

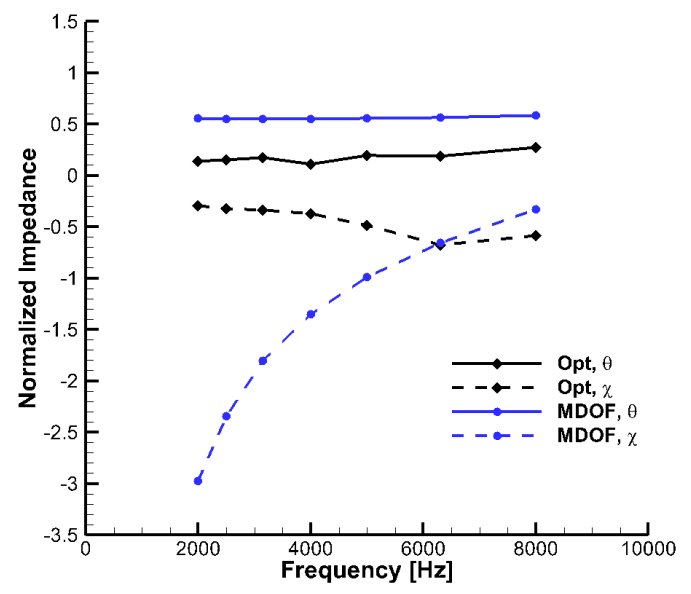

(b) Hub Liner - 2.

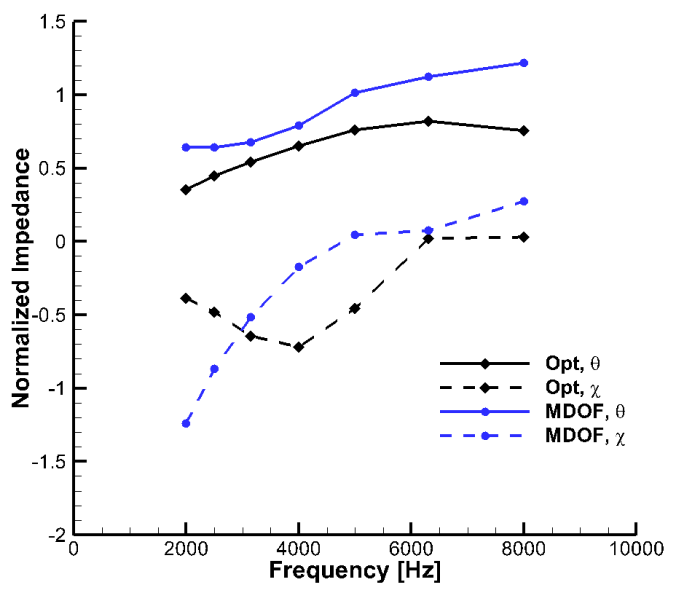

(c) Nacelle Liner.

Figure 5: Comparison of optimum and design impedance spectra. 


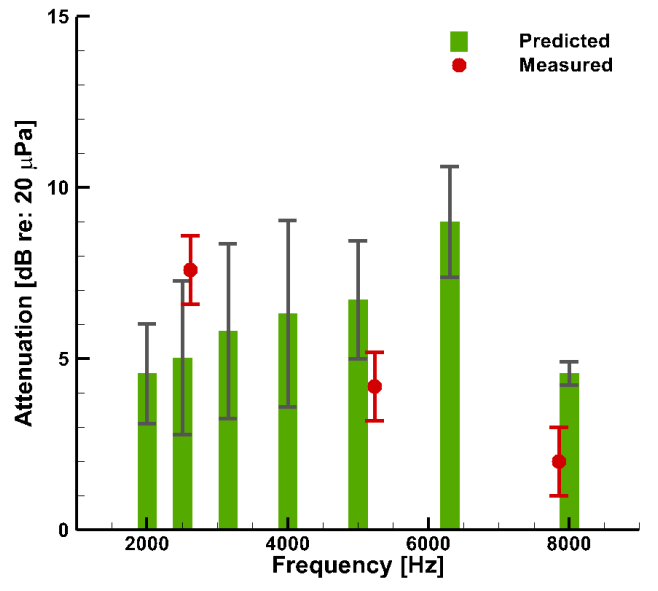

(a) $61 \%$ Fan RPMc.

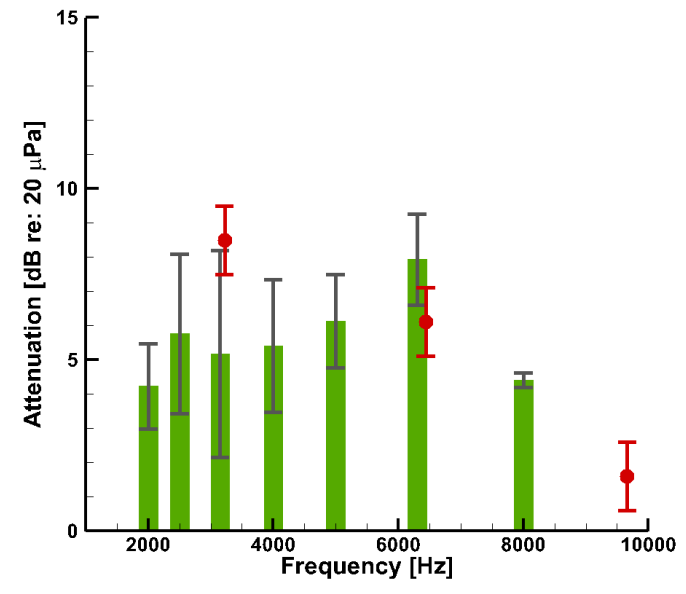

(b) $75 \%$ Fan RPMc.

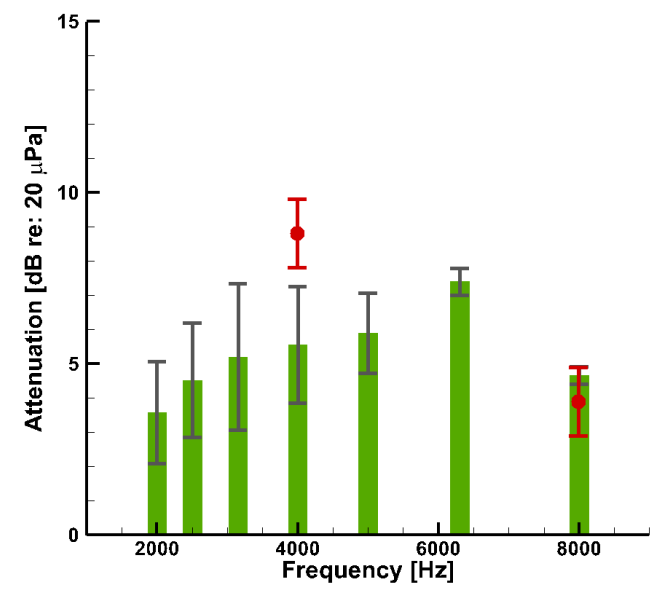

(c) $93 \%$ Fan RPMc.

Figure 6: Comparison of predicted and measured in-duct attenuation values for the single-degree of freedom (SDOF) liner. Error bars indicate estimated $95 \%$ confidence intervals on the predicted values and expected error of $+/-1 \mathrm{~dB}$ on the measured values. 


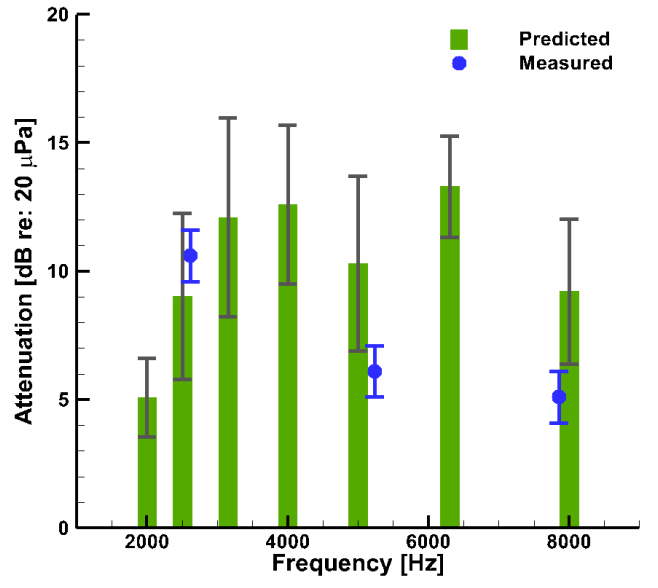

(a) $61 \%$ Fan RPMc.

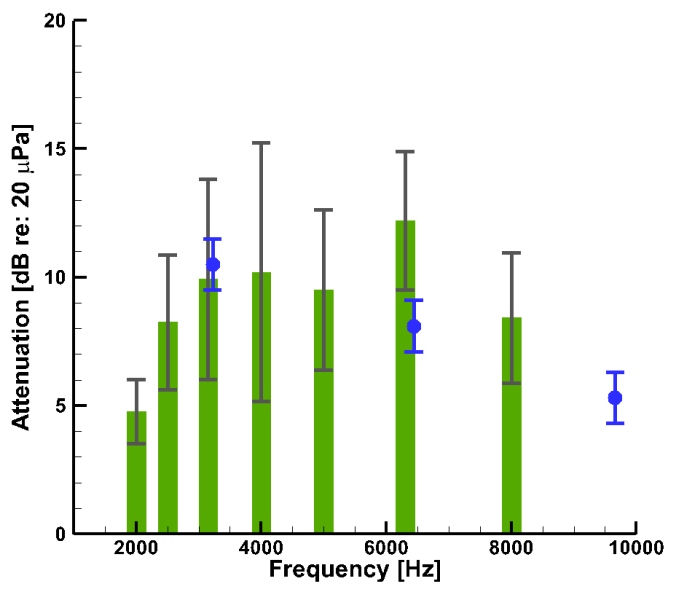

(b) $75 \%$ Fan RPMc.

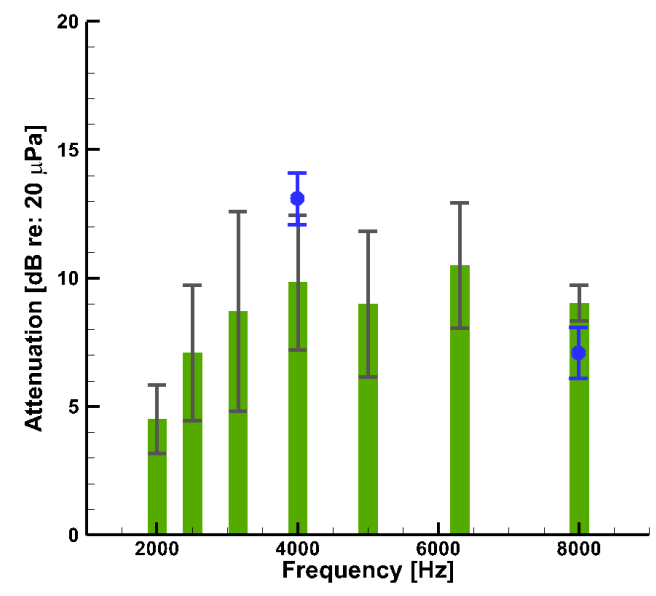

(c) $93 \%$ Fan RPMc.

Figure 7: Comparison of predicted and measured in-duct attenuation values for the multi-degree of freedom (MDOF) liner. Error bars indicate estimated $95 \%$ confidence intervals on the predicted values and expected error of $+/-1 \mathrm{~dB}$ on the measured values. 